\title{
Relationships of Demographic Background and Practice Setting Among Practicing Physician Assistants in the United States
}

\author{
Justin Kelley *, Shawn Lies *, Richard Muma \\ Department of Physician Assistant - College of Health Professions
}

\begin{abstract}
The assumption has always been that minority health care providers were more likely to work in areas of need. However, no recent research has been conducted to determine this. The purpose of this study was to determine the relationships between current practice specialty and population served to demographic variables (e.g., race) among physician assistants (PAs). This cross-sectional study measured demographic and practice setting survey data. A random sampling of 10,500 PAs was surveyed. Nearly $12 \%$ of the sample was minority (compared to nearly $25 \%$ nationally). The main results indicated minority PAs were more likely to work in underserved and primary care practices as compared to non-minorities (Underserved $=31.9 \%$ vs. $19.3 \%$; Primary Care $=38.8 \%$ vs. $29.3 \%$ ). Separately, there was a significant relationship among all those serving underserved populations and non-married individuals and those over age 39 (more likely to serve in this setting) $(\mathrm{p}<0.01)$. Household income less than $\$ 50,000$ at the time of high school graduation was significantly related to serving underserved and primary care populations (more likely to serve in these settings) $(\mathrm{p}<0.01 ; \mathrm{p}<0.001)$. In this sample minority PAs were more likely to serve in underserved and primary care settings. Certain demographics among all respondents were also significantly related to service in underserved and primary care settings.
\end{abstract}

\section{Introduction}

In 2000, the minority population in the United States was estimated to be $24.9 \%$ by the U.S. Census Bureau [1]. Minority representation in the physician profession was $10.2 \%$ [2], while minority representation in the physician assistant profession was $16.7 \%$ [3]. According to a 1985 study, underrepresented minority physicians were more likely to care for minority patients than White physicians [4]. A significant disparity in providers of health care to the minority population exists. Increasing the number of providers caring for the underserved will impact access to medical care significantly, as well as likely having an impact on the overall cost and quality of health care. If demographic predictors could be found to identify these providers, more efficient policies to increase their numbers could be implemented. Efforts have been made on a national level to increase the recruitment, retention and academic success of underrepresented minorities in health fields.

As basis for comparison to our study, a 1993 study of the physician profession demonstrated that variables found to be predictive $(\mathrm{p}<0.001)$ of providing substantial care to underserved populations included whether the physician was a member of an underserved minority, had participated in the National Health Service Corps, had a strong interest in practicing in an underserved area before medical school, and grew up in an underserved area [5].

No recent research on a national level has been conducted to verify that minority physician assistants are more likely to work in areas of need. The purpose of this study was to determine significant relationships between the demographic background of practicing physician assistants and their current practice settings. Likewise, we attempted to determine whether current practicing minority physician assistants returned to communities serving minority populations after attending an accredited physician assistant program.

\section{Experiment, Results, Discussion, and Significance}

This study was a cross-sectional, random sampling of PAs from the United States. The research question was as follows:

- What were the relationships between current practice specialty and population served to demographic variables among practicing PAs in the United States?

The American Academy of Physician Assistants (AAPA) database was queried to obtain a randomized sample of PAs in the United States. 10,500 PAs were surveyed to ensure a pool of respondents of approximately 2,000. A 
survey was mailed asking a series of questions in regard to current practice specialty (e.g., primary care, specialty, academician, not in clinical practice), population served (e.g., work setting designated as a facility that serves an underserved population or community), current practice community size (urban $=>50,000$; rural $=<50,000$ ), PA degree level (certificate, associate, bachelor, or master), relationship status (married or not married), gender, age, race/ethnicity, primary language (English, Spanish, or other language), family size (size of your immediate family at the time of your high school graduation) and income during high school, high school classification (public or private) and High School and PA program GPA.

Frequency counts were conducted to determine current practice specialty, population served, current practice community size, PA degree level, relationship status, gender, age, race/ethnicity, language, family size and income during high school, high school classification and GPA, and PA program GPA. Chi-square analyses were performed to determine whether there were any significant relationships between current practice specialty and population served to the demographic variables noted above. Surveys were collected from May 11, 2008, to July 4, 2008 , with a $20 \%(n=2048)$ response rate.

Nearly $12 \%$ of the sample was minority (compared to nearly $25 \%$ nationally). The main results indicated minority PAs were more likely to work in underserved and primary care practices as compared to non-minorities (Underserved $=31.9 \%$ vs. $19.3 \%$; Primary Care $=38.8 \%$ vs. $29.3 \%$ ). Separately, there was a significant relationship among all those serving underserved populations and non-married individuals and those over age 39 (more likely to serve in this setting) $(\mathrm{p}<0.01)$. Household income less than $\$ 50,000$ at the time of high school graduation was significantly related to serving both underserved and primary care populations (more likely to serve in these settings) $(\mathrm{p}<0.01 ; \mathrm{p}<0.001)$.

This was a nationwide survey with an adequate response rate; in which generalizations can be made. The focus of the study was to obtain a current and general understanding of the PA population and the relationship of demographic background and practice setting. The significance of this study rests in the fact that no recent study has been conducted that surveyed PAs on a national level to determine relationships between demographic information and their current practice setting.

Given these results, future research may be aimed at different methods of recruitment needed to increase the percentage of minority applicants to physician assistant programs. Increasing diversity could ultimately assist in resolving health care disparities by placing a higher percentage of PAs in underserved populations.

\section{Conclusions}

The results of this study demonstrate that minority PAs practice in underserved, primary care settings compared to non-minority PAs. Certain demographics, including non-married status, age over 39, and household income less than $\$ 50,000$ at time of high school graduation, among all respondents were significantly related to service in underserved and primary care settings.

\section{Acknowledgements}

We would like to thank Richard Muma, PhD, MPH, PA-C, Research Advisor. Permission was granted by Dr. Muma to use previous survey suggestions from Angela Armour and Robin Williamson, in their 2008 project entitled Factors Influencing Rural Physician Assistant Practice. This project was funded in part by the Workforce Diversity Initiative Grant, funded by US/DHHS, grant number D57HP05123, 2005-2008, Richard Muma, PI.

\section{References}

[1] United States Census Bureau. Census 2000. 2000.

[2] Association of Medical Colleges: Diversity in the Physician Assistant Workforce: Facts \& Figures 2006. 2006:126.

[3] AAPA: AAPA Physician Assistant Census Report. 2007: Table 1.3.

[4] Keith SN BR, Swanson AG, Williams AP. Effects of Affirmative Action in Medical Schools: A Study of the Class of 1975. New England Journal of Medicine. 1985;313:1519-1525.

[5] Rabinowitz HK, Diamond JJ, Veloski JJ, Gayle JA. The Impact of Multiple Predictors on Generalist Physicians' Care of Underserved Populations. American Journal of Public Health. 2000:90(8):1225-1228. 